\title{
sTREM-1 in bronchoalveolar lavage fluid in patients with pulmonary sarcoidosis, effect of smoking and inflammation
}

\author{
Suchankova $\mathrm{M}^{1}$, Bucova $\mathrm{M}^{1}$, Tibenska $\mathrm{E}^{2}$, Demian $\mathrm{J}^{3}$, Majer $\mathrm{I}^{3}$, Novosadova $\mathrm{H}^{3}$, Tedlova $\mathrm{E}^{3}$, \\ Durmanova $\mathrm{V}^{1}$, Paulovicova $\mathrm{E}^{4}$
}

Institute of Immunology, Faculty of Medicine Comenius University Bratislava, Slovakia.
maria.bucova@fmed.uniba.sk

\begin{abstract}
Soluble TREM-1 (sTREM-1; Triggering receptor expressed on myelocytes) is a new inflammatory marker indicating the intensity of myeloid cells activation and the presence of infection caused by extracellular bacteria and mould.

The aim of our work was to detect and compare the levels of sTREM-1 in bronchoalveolar lavage fluid (BALF) in patients with pulmonary sarcoidosis (PS) and other ILD of non-infectious origin. The STREM-1 levels were assessed by ELISA in 46 patients suffering from ILD, out of them 22 with PS.

The levels of BALF STREM-1 in PS patients were higher than in control group of ILD patients of non-infectious origin, however, the difference was not statistically significant. Since all PS patients except one were nonsmokers we compared non-smokers PS with non-smokers ILD patients and found four times higher levels of BALF sTREM-1 in PS patients $(P=0.001)$. We also recorded the effect of smoking, ILD smokers had higher sTREM-1 levels than non-smokers $(P=0.0019)$. Higher concentrations of sTREM-1 were detected in BALF of patients with lymphadenopathy and with elevated inflammatory markers in BALF.

Our results show that BALF STREM-1 could be a good inflammatory marker and could help in diagnosis and PS monitoring. Detection of STREM-1 in BALF indirectly points to myeloid cells activation in the lungs and helps to complete the information about the number of myeloid cells commonly determined in BALF with additional information concerning the intensity of their activation. This is the first study that analyses BALF STREM-1 levels in patients with PS (Tab. 8, Ref. 28). Text in PDF www.elis.sk.

Key words: bronchoalveolar lavage fluid, inflammation, interstitial lung diseases, sarcoidosis, TREM-1.
\end{abstract}

TREM-1 (Triggering receptor expressed on myeloid cells), first described in 2000, is expressed on the surface of myeloid cells (1) and its expression is further enhanced by an inflammatory process. Myeloid cells activation upon a ligand binding to this receptor generally results in amplification of inflammatory responses. Macrophages are converted into activated cells producing high levels of proinflammatory cytokines and chemokines attracting further cells to the site of inflammation (1-3). However, anti-inflammatory cytokine production upon TREM-1 activation is decreased (2). Another consequence of TREM-1 activation is an increased expression of co-stimulatory molecules $(1,2)$ on the surface of mac-

${ }^{1}$ Institute of Immunology, Faculty of Medicine Comenius University Bratislava, Slovakia ${ }^{2}$ Medirex ltd, Bratislava, Slovakia, ${ }^{3}$ Department of Pneumology and Phthisiology, Faculty of Medicine Comenius University, Bratislava, Slovakia, and ${ }^{4}$ Institute of Chemistry, Slovak Academy of Science, Centre of Excellence Glycomed, Bratislava, Slovakia

Address for correspondence: M. Bucova, MD, PhD, Institute of Immunology, Faculty of Medicine Comenius University, Spitalska 14, SK-813 72 Bratislava, Slovakia.

Phone: +421.2.59357351, Fax: +421.2.59357578

Acknowledgements: This work was supported by the grant VEGA $1 / 0574 / 10$. This contribution is the result of project implementation: Centre of Excellence for Glycomics, ITMS 26240120031, supported by the Research \& Development Operational Programme funded by ERDF. rophages, and enhanced antigen presentation that triggers a dramatic T cell proliferation. Neutrophils activated through TREM-1 enhance their respiratory burst activity, degranulation, phagocytosis, release of myeloperoxidase and IL-8, respectively (4). Although the biochemical structure of TREM-1 ligands remains to be identified, some studies suggest inflammatory response products participation (5-7). Expression of membrane bound TREM-1 on myeloid cells is enhanced in the presence of bacteria and bacterial products, inflammatory cytokines and TREM-1 activation $(2,3)$. However, anti-inflammatory cytokines synergistically inhibit their expression (2). The membrane form of TREM-1 can be cleaved by plasma metalloproteinases and its ectodomain is released into microenvironment (8). This soluble TREM-1 molecule (sTREM-1) could act as a down-regulator of inflammation - by binding to the natural ligand, sTREM-1 prevents its binding to the membrane TREM-1 and subsequent cell activation (9).

STREM-1 can be detected in plasma $(8,10-12)$, pleural effusion $(13,14)$, amniotic fluid (15), bronchoalveolar lavage $(16,17)$ and other body fluids. Several studies have tried to elucidate the biological significance of TREM-1 receptor and its soluble form in various diseases and in various forms of inflammatory response, e.g. sepsis, autoimmune diseases and allergies. The highest expression of TREM-1 and levels of sTREM-1 molecules have been found in patients with sepsis (10) and other inflammatory diseases caused by 
extracellular microorganisms such as pneumonia $(16,17)$, cholangitis $(18,19)$ and acute pancreatitis $(20)$. Less significant involvement of this molecule but still higher compared to healthy controls was found in patients with chronic obstructive pulmonary disease (COPD) (11) and inflammatory bowel diseases (12). There are only a few studies testing TREM-1 in diseases affecting the lung interstitium - interstitial lung diseases (ILD) and no study analyzing BALF sTREM-1 in patients with pulmonary sarcoidosis (PS).

The aim of our study was to detect and compare the levels of a new inflammatory marker STREM-1 in BALF of patients with PS and control group of ILD patients of non-infectious origin.

\section{Patients and methods}

51 patients indicated by clinicians for BALF examination for diagnostic purposes were enrolled to our study. Five patients were excluded from the study for the following reasons: two due to bronchopneumonia, two due to pulmonary neoplasia and one due to pancytopenia. Finally, 46 patients were enrolled in this study, 22 with PS and 24 ILD patients of non-infectious origin (Tab. 1). Whereas the BAL procedure is an invasive examination and there is an ethical problem to enroll healthy individuals, the group of ILD patients other than PS were used as a control cohort in our study. The disadvantage of our control group is that it is heterogeneous group of different clinical entities and increased expression of TREM-1 can be caused by infection (e.g. in patients with chronic obstructive pulmonary disease acompanied by chronic infection). The comparison of sarcoidosis and other ILD may be interesting in the differential diagnosis. The diagnosis was established in compliance with criteria recommended by the American Thoracic Society (ATS), the European Respiratory Society (ERS) and the World Association of Sarcoidosis and Other Granulomatous Disorders (WASOG) (21): typical clinico-radiological presentation, the histological evidence of non-caseating granuloma and/or an increased CD4/CD8 ratio (>3.5) in BALF. According to chest radiographic staging, 8 patients were in stage I of the disease and 14 patients in stage II. Other possible diseases causing granuloma were excluded.

The control group represented 24 patients suffering from ILD of non-infectious origin, more unspecified interstitial pneumopathy with non-infectious chronic interstitial lung involvement. Inclusion criteria for this group were confirmed signs of fibrosis on HRCT scan - GGO (ground-glass opacity) and/or honeycombing (all patients), decreased lung diffusion capacity (all patients), typical BAL pattern - decreased CD4/CD8 ratio (all patients).

Both groups were further subdivided into subgroups of smokers and non-smokers, patients with and without lymphadenopathy, patients with and without elevated inflammatory parameters in BALF (total proteins, immunoglobulins, complement compo- nents, alpha 2 -macroglobulin) (Table 6). No patient enrolled in this study was treated with corticosteroids prior to BAL procedure. The study was approved by the Local Ethical Committee of Faculty of Medicine Comenius University in Bratislava and written informed consent was obtained from all patients.

BALF harvesting during bronchoscopy was carried out at the Department of Pneumology and Phthisiology, Faculty of Medicine Comenius University in Bratislava. Fiberoptic bronchoscope was introduced into the wedge position to middle lobe bronchus to the segment S4 or S5. BAL procedure was BALF withdrawing performed by instillation of $100 \mathrm{ml}$ in two successive $50 \mathrm{ml}$ portions of sterile normal saline into the middle lobe and aspirated by gentle suction using a flexible fiberoptic bronchoscope. The harvested BALF was split equally into three tubes. One tube was sent for the standard analysis of the differential cell count (Table 7) and immunological tests (Table 6), next one was transported to the laboratory of clinical microbiology (cultivation of aerobic, anaerobic bacteria and mycobacterium tuberculosis to rule out infection) and the third tube was placed on ice and transported to our laboratory, where the sample was filtered through sterile mull and centrifuged at $400 \mathrm{~g}$ for $10 \mathrm{~min}$ at $+4{ }^{\circ} \mathrm{C}$. Thereafter, the sample was stored at $-80{ }^{\circ} \mathrm{C}$ until the assays were performed. Plasma of patients was obtained from whole blood taken into a tube with EDTA after centrifugation at $400 \mathrm{~g}$ for $15 \mathrm{~min}$.

sTREM-1 concentrations in BALF were determined by a sandwich enzyme-linked immunosorbent assay (Quantikine, Human TREM-1 ELISA kit, R\&D Systems, USA) according to the instructions of the manufacturer.

\section{Statistical analysis}

The one-sample Kolmogorov-Smirnov test was used to determine whether the investigated population followed a normal distribution. On the basis of the results, either non-parametric Mann-Whitney U-test or the parametric non-paired $t$-test with Welch correction was used to examine the statistical significance. Data with determined cut off were compared with Fischer's test (comparison of inflammatory markers). To calculate correlation between two sets of data, Pearson (data followed normal distribution) or Spearman's (data did not follow normal distribution) test was used.

\section{Results}

\section{Plasma levels of STREM-1}

In a small cohort of patients with PS $(n=7)$ and healthy controls $(n=19)$ we tested the plasma concentrations of sTREM-1 and found no statistically significant difference between these two groups (Tab. 1).

Tab. 1. Concentrations of sTREM-1 in plasma of patients with pulmonary sarcoidosis and healthy controls.

\begin{tabular}{lcccc}
\hline & \multicolumn{2}{c}{ sTREM-1 in plasma (pg/ml) } & Mann-Whitney & Range \\
\cline { 2 - 4 } Group & Number & Mean \pm SD & Median & 2-tailed, $\mathrm{p}=$ \\
PS & 7 & $24.80 \pm 17.73$ & 18.83 & $9.67-55.04$ \\
Healthy controls & 19 & $26.16 \pm 6.06$ & 26.59 & $15.98-38.78$ \\
\hline
\end{tabular}

sTREM-1 - soluble triggering receptor expressed on myelocytes, PS - pulmonary sarcoidosis. 
Tab. 2. Characteristics of study subjects.

\begin{tabular}{lccc}
\hline Group & Subjects number & Male $(\%)$ & Mean age \pm SD \\
\hline PS & 22 & 81.8 & $38.4 \pm 10.7$ \\
ILD & 24 & 54.2 & $57.0 \pm 14.1$ \\
Non-smokers PS & 21 & 78.6 & $38.6 \pm 10.9$ \\
Smokers PS & 1 & 100.0 & 32.0 \\
Non-smokers ILD & 15 & 46.2 & $58.9 \pm 16.5$ \\
Smokers ILD & 9 & 66.7 & $56.3 \pm 8.6$ \\
Patients without LAP & 18 & 58.3 & $60.7 \pm 15.9$ \\
Patients with LAP & 28 & 82.1 & $41.7 \pm 12.14$ \\
BALF INFLAM + & 19 & 78.6 & $38.5 \pm 10.4$ \\
BALF INFLAM - & 27 & 56.5 & $51.9 \pm 16.3$ \\
\hline
\end{tabular}

PS - pulmonary sarcoidosis, ILD - interstitial lung diseases, LAP - lymphadenopathy, BALF INFLAM+ - elevated inflammatory markers in bronchoalveolar lavage fluid with elevated levels of at least 3 inflammatory markers, BALF INFLAM- not-elevated inflammatory markers in bronchoalveolar lavage fluid.

Tab. 3. ILD and subgroups of smokers, non-smokers, LAP positive/ negative and BALF inflammatory markers positive/negative patients.

\begin{tabular}{llccc}
\hline ILD & Smokers & Non-smokers & LAP +/- & BALF INFLAM +/- \\
\hline IPF & $5(3$ COPD) & 11 & $3 / 13$ & $7 / 9$ \\
CT ILD & 2 (1 COPD) & 3 & $1 / 4$ & $1 / 4$ \\
EAA & 2 & 0 & $2 / 0$ & $2 / 0$ \\
DI ILD & 0 & 1 & $0 / 1$ & $0 / 1$ \\
\hline All patients & $\mathbf{9}$ & $\mathbf{1 5}$ & $\mathbf{6} / \mathbf{1 8}$ & $\mathbf{1 0} / \mathbf{1 4}$
\end{tabular}

ILD - interstitial lung diseases, LAP +/- - number of patients with lymphadenopathy/ number of patients without lymphadenopathy, BALF INFLAM+/- - number of patients with elevated inflammatory markers in bronchoalveolar lavage fluid/ number of patients without elevated inflammatory markers in bronchoalveolar lavage fluid, IPF - idiopathic pulmonary fibrosis, COPD - chronic obstructive pulmonary disease, CT ILD - connective tissue related interstitial lung disease, EAA - extrinsic allergic alveolitis, DI ILD - drug-induced interstitial lung disease.

\section{Characteristics of the study groups (PS and ILD patients)}

Plasma levels of sTREM-1 could not indicate inflammatory process ongoing in lungs that is why we decided to test the levels of sTREM-1 in BALF of PS patients. Since bronchoalveolar la- vage is not performed in healthy subjects due to ethical reasons, as comparative control group were chosen patients suffering from other interstitial lung diseases (ILD) of non-infectious origin. Characteristics of study subjects are shown in Table 2 and Table 3.

sTREM-1 in BALF of PS and ILD patients. The effect of smoking.

Comparing levels of STREM-1 in BALF of patients with PS $(n=22)$ and the rest of ILD patients of non-infectious origin $(n=24)$ we found higher mean value in patients with PS compared to patients with other ILD (PS: mean \pm SD: $265.01 \pm 170.40$, ILD: mean \pm SD: $189.40 \pm 158.03)$, however, the difference was not statistically significant $(\mathrm{p}=0.125)$ (Tab. 4).

The BALF levels of sTREM-1 in PS non-smokers $(n=21$; only one patients in this group was smoker) were more than four times higher than in non-smokers in the control ILD group (PS: median: 267.8; range: 34.41-565.90; ILD: median: 30; range: 15.20-267.04). The difference was statistically highly significant $(\mathrm{p}=0.001)$ (Tab. 4).

Comparing ILD smokers ( $\mathrm{n}=9 ; 4$ of these patients suffered also from COPD and 2 from bullous emphysema) and non-smokers $(n=13)$, we found statistically significantly higher levels of BALF sTREM-1 in ILD smokers (ILD smokers: median: 331.8, range: 133.24-512.97; ILD non-smokers (median: 30, range: 15.20-267.04). This difference was statistically highly significant $(\mathrm{p}=0.0019)$ (Tab. 4).

\section{Levels of BALF sTREM-1. The effect of inflammation.}

As high concentrations of sTREM-1 suggest the amplification of inflammation, we compared sTREM-1 levels in patients with and without hilar and/or mediastinal lymphadenopathy (LAP), regardless of the diagnosis. Higher levels of BALF sTREM-1 were found in patients with LAP (median: 260.3, range: 24.40-565.9)

Tab. 4. Concentrations of sTREM-1 in BALF of patient with pulmonary sarcoidosis and other interstitial lung diseases and influence of smoking.

\begin{tabular}{|c|c|c|c|c|c|}
\hline \multirow[b]{2}{*}{ Group } & \multicolumn{5}{|c|}{ sTREM-1 in BALF (pg/ml) } \\
\hline & Number & Mean \pm SD & Median & Range & $\mathrm{p}=$ \\
\hline$\overline{\mathrm{PS}}$ & 22 & $265.01 \pm 170.40$ & 243.94 & $34.41-565.90$ & \multirow{2}{*}{$0.125^{*}$} \\
\hline ILD & 24 & $189.40 \pm 158.03$ & 181.65 & $15.20-512.97$ & \\
\hline Smokers PS & 1 & 218.90 & 218.90 & & \\
\hline Non-smokers PS & 21 & $276.70 \pm 185.10$ & 267.76 & $34.41-565.90$ & - \\
\hline Non-smokers PS & 21 & $276.70 \pm 185.10$ & 267.76 & $34.41-565.90$ & \multirow{2}{*}{$0.001 * *$} \\
\hline Non-smokers ILD & 15 & $94.90 \pm 99.70$ & 30.00 & $15.20-267.04$ & \\
\hline Non-smokers ILD & 15 & $94.90 \pm 99.70$ & $\mathbf{3 0 . 0 0}$ & $15.20-267.04$ & \multirow{2}{*}{$0.0019 * *$} \\
\hline smokers ILD & 9 & $319.80 \pm 150.70$ & 331.82 & 133.24-512.97 & \\
\hline
\end{tabular}

sTREM-1 - soluble triggering receptor expressed on myelocytes, BALF - bronchoalveolar lavage fluid, PS - pulmonary sarcoidosis, ILD - interstitial lung diseases, * - t-test

2-tailed, ** - Mann-Whitney test 2-tailed.

Tab. 5. Concentrations of sTREM-1 in BALF of patients with LAP and patients with increased inflammatory markers in BALF irrespective of diagnosis.

\begin{tabular}{|c|c|c|c|c|c|}
\hline Group (PS+ILD) & $\mathrm{N}(\mathrm{PS}+\mathrm{ILD})$ & Mean \pm SD & Median & Range & $\mathrm{p}=$ \\
\hline Patients with LAP & $22+6$ & $267.20 \pm 165.50$ & 260.29 & $24.40-565.9$ & \multirow{2}{*}{$0.0064 * *$} \\
\hline Patients without LAP $(\mathrm{N}=18)$ & $0+18$ & $127.60 \pm 149.00$ & 31.11 & $15.20-449.50$ & \\
\hline 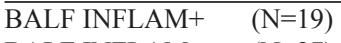 & $9+10$ & $283.17 \pm 191.24$ & 260.29 & $15.20-565.90$ & \multirow{2}{*}{$0.042 *$} \\
\hline BALF INFLAM- & $13+14$ & $166.82 \pm 142.00$ & 135.44 & $18.20-512.97$ & \\
\hline
\end{tabular}

sTREM-1 - soluble triggering receptor expressed on myelocytes, BALF - bronchoalveolar lavage fluid, PS - pulmonary sarcoidosis, ILD - interstitial lung diseases, LAP - lymphadenopathy, BALF INFLAM+ - elevated inflammatory markers in bronchoalveolar lavage fluid, BALF INFLAM- - non-elevated inflammatory markers in bronchoalveolar lavage fluid, N(PS+ILD) - number of patients with PS + number of patients with ILD, * - t-test 2-tailed, ** - Mann-Whitney test 2-tailed. 
Tab. 6. Inflammatory markers in BALF of patients with pulmonary sarcoidosis and interstitial lung diseases.

\begin{tabular}{|c|c|c|c|c|c|}
\hline & \multicolumn{2}{|r|}{ PS } & \multicolumn{2}{|r|}{ ILD } & \multirow{2}{*}{$\mathrm{p}=$} \\
\hline & Mean \pm SD & No. of inflam+patients/cut off (mg/l) & Mean \pm SD & No. of inflam+patients/cut off $(\mathrm{mg} / \mathrm{l})$ & \\
\hline IgG & $53.10 \pm 68.50$ & $18 />30.0$ & $55.50 \pm 102.30$ & $13 />30.0$ & $0.53 *$ \\
\hline $\operatorname{IgM}$ & - & $11 />2.4$ & - & $11 />2.4$ & $0.93 * *$ \\
\hline $\operatorname{Ig} A$ & $17.20 \pm 21.40$ & $7 />20.0$ & $20.40 \pm 31.80$ & $11 />20.0$ & $0.98^{*}$ \\
\hline Total proteins & $266.4 \pm 245.7$ & $18 />200$ & $243.30 \pm 216.10$ & $16 />200$ & $0.99 *$ \\
\hline $\mathrm{C} 3$ & - & $9 />2.5$ & - & $7 />2.5$ & $0.69 * *$ \\
\hline A2MA & - & $7 />4.0$ & - & $7 />4.0$ & $1.00 * *$ \\
\hline
\end{tabular}

$\mathrm{BALF}$ - bronchoalveolar lavage fluid, No. of inflam+ patients/cut off - number of patients with elevated marker (higher than the determined cut off value)/determined cut off value; PS - pulmonary sarcoidosis, ILD - interstitial lung diseases, IgG - immunoglobulin G, IgM - immunoglobulin M, IgA - immunoglobulin A, C3 - C3 complement component, A2MA - alpha 2-macroglobulin, * - Mann-Whitney test, ** - Fischer's test.

Tab. 7. CD surface markers and differential cell count in BALF.

\begin{tabular}{lccc}
\hline & $\begin{array}{c}\text { PS } \\
(\text { mean } \pm \text { SD) }\end{array}$ & $\begin{array}{c}\text { ILD } \\
(\text { mean } \pm \text { SD) }\end{array}$ & $\begin{array}{c}\mathrm{p}= \\
\text { (Mann-Whitney, } \\
\text { 2-tailed sg.) }\end{array}$ \\
\hline CD3+ (\%) & $93.20 \pm 4.10$ & $89.50 \pm 5.40$ & $=0.0052$ \\
CD4+ (\%) & $78.40 \pm 16.10$ & $37.70 \pm 13.20$ & $<0.0001$ \\
CD8+ (\%) & $15.70 \pm 14.50$ & $53.90 \pm 14.50$ & $<0.0001$ \\
CD4+/CD8+ & $8.60 \pm 5.80$ & $0.80 \pm 0.50$ & $<0.0001$ \\
Ma (\%) & $53.50 \pm 17.10$ & $68.30 \pm 20.10$ & $=0.0104$ \\
Ly (\%) & $35.80 \pm 16.90$ & $16.20 \pm 12.20$ & $=0.0001$ \\
Neu (\%) & $10.10 \pm 8.30$ & $14.80 \pm 13.30$ & $=0.4$ \\
Eo (\%) & $0.60 \pm 0.90$ & $0.70 \pm 1.50$ & $=0.75$ \\
\hline CD $\%$.
\end{tabular}

$\mathrm{CD}$ - cluster of differentiation - cell membrane markers, BALF - bronchoalveolar lavage fluid, PS - pulmonary sarcoidosis, ILD - interstitial lung diseases, CD3+percentage of all T-lymphocytes, CD4+- percentage of T-helper lymphocytes, CD8+ - percentage of T-cytotoxic lymphocytes, CD4/CD8 - ratio between T-helper and T-cytotoxic lymphocytes percentage, $\mathrm{Ma}$ - macrophages, Ly - lymphocytes, Neu neutrophils, Eo - eosinophils.

Tab. 8. Correlation between sTREM-1 concentration and percentage of cells in BALF.

\begin{tabular}{lccc}
\hline Cells/sTREM-1 & $\mathrm{R}=$ & $95 \%$ CI & $\mathrm{p}=$ \\
\hline MA/sTREM-1 & -0.310 & $-0.662-0.154$ & 0.172 \\
LY/sTREM-1 & 0.246 & $-0.221-0.621$ & 0.284 \\
NEU/sTREM-1 & -0.123 & $-0.537-0.338$ & 0.595 \\
CD4+/sTREM-1 & 0.452 & $0.012-0.746$ & $\mathbf{0 . 0 3 9 6}$ \\
CD8+/sTREM-1 & -0.289 & $-0.641-0.165$ & 0.193 \\
CD4/CD8/sTREM-1 & 0.325 & $-0.126-0.664$ & 0.140 \\
\hline
\end{tabular}

Cells/sTREM-1 - correlation between selected cells and sTREM-1, Ma - macrophages, Ly - lymphocytes, Neu - neutrophils, Eo - eosinophils, CD4+ - T-helper lymphocytes, CD8+- T-cytotoxic lymphocytes, CD4/CD8 - ratio between T-helper and T-cytotoxic lymphocytes, $\mathrm{R}$ - correlation coefficient, $\mathrm{CI}$ - confidence interval

than in patients without LAP (median: 31.11 , range: $15.20-449.50$ ) $(\mathrm{p}=0.0064)$ (Tab. 5).

Moreover, patients with at least 3 elevated inflammatory markers (total proteins, IgG, IgA, IgM, C3 complement, alfa-2 macroglobulin) in BALF (sTREM-1: mean \pm SD: $283.17 \pm 191.24$ ) compared to patients without elevated inflammatory markers (sTREM-1: mean \pm SD: $166.82 \pm 142$ ) showed statistically significantly higher levels of sTREM-1 in BALF ( $\mathrm{p}=0.042$ ), regardless of the diagnosis (Tab. 5). Concentrations of investigated inflammatory markers (proteins, IgG, IgA, IgM, C3, alfa-2 macroglobulin) in BALF did not differ between PS and ILD investigated groups (Tab. 6).

\section{Differential cell count in BALF of PS and ILD patients}

Comparing both investigated groups, the group of PS patients had statistically significantly higher percentage of all lymphocytes (mean \pm SD: PS: $35.80 \pm 16.90$; ILD: $16.20 \pm 12.20$; $\mathrm{p}=0.0001$ ), higher percentage of $\mathrm{CD}^{+} \mathrm{T}$ cells (PS: $93.20 \pm 4.10$; ILD: 89.50 \pm 5.4 ; $\mathrm{p}=0.0052$ ), mainly $\mathrm{CD}^{+}$helper T cells (PS: $78.40 \pm 16.10$; ILD: $37.70 \pm 13.20 ; \mathrm{p}<0.0001$ ) and $\mathrm{CD}^{+} / \mathrm{CD}^{+}$ratio (PS: $8.60 \pm 5.80$; ILD: $0.80 \pm 0.50 ; \mathrm{p}<0.0001$ ) (Tab. 7).

Very interesting is the significant correlation between the levels of sTREM-1 in BALF and the percentage of $\mathrm{CD}^{+} \mathrm{T}$ cells in BALF of PS patients (Spearman non parametric 2 - tailed test: $\mathrm{p}=0.0396$; Spearman $\mathrm{R}=0.452,95 \% \mathrm{CI}=0.012-0.746)$ (Tab. 8).

None of the PS patients had positive cultivation for aerobic, anaerobic bacteria and mycobacterium tuberculosis.

\section{Discussion}

Sarcoidosis is a multisystemic disease of unknown origin, characterized by the formation of granulomatous lesions especially in the lungs or the lymph nodes, but virtually any organ can be affected. Sarcoidosis is a disease of unknown etiology that is classified within a group of about 200 diseases termedinterstitial lung diseases (ILD; e.g. idiopathic pulmonary fibrosis, hypersensitivity pneumonitis, drug-induced ILD, radiation-induced ILD, sarcoidosis, eosinophilic pneumonias, connective tissue disease - related ILD and many other disorders (22). However, in comparison with other ILD, sarcoidosis has some specificities. For the acute stage lymphadenopathy (LAP), lymphocytic alveolitis with $\mathrm{T}$ helper lymphocytes (CD4+) predominance (unlike other ILD) is typical indicating a strong activation of macrophages and intensive antigen presentation. The antigen responsible for induction of such immune response in patients with sarcoidosis is not yet known. Potential disease-causing agent according to recent studies is mold $(23,24)$.

It is known that increased expression of TREM-1 on the surface of myeloid cells refers to the presence of extracellular microorganisms (bacteria or fungi) $(2,16,25,26)$. This membrane form of TREM-1 can be cleaved and its soluble form sTREM-1 is detectable in plasma and other biological fluids. In our study we wanted originally to compare the plasma levels of sTREM-1 in patients with pulmonary sarcoidosis and healthy controls. In a pilote study with a small number of subjects we found no differences in plasma concentrations of PS patients and healthy subjects. We realised that plasma levels of sTREM-1 do not indicatean ongoing inflammatory process in lungs that is why we decided to test the levels of sTREM-1 directly in BALF. Since bronchoalveolar lavage is not 
performed in healthy subjects for ethical reasons, as comparative control group patients suffering from other interstitial lung diseases (ILD) of non-infectious origin were chosen.

Our results showed that the levels of sTREM-1 in BALF of PS patients were higher than in control group of ILD patients of noninfectious origin (PS: mean \pm SD: $265.01 \pm 170.40$, ILD: mean \pm SD: $189.40 \pm 158.03)$, however, the difference did not reach statistical significance (Tab. 4).

Since all PS patients except one were non-smokers (what is typical for PS patients) we wanted to test the influence of smoking to BALF sTREM-1 levels (Tab. 4). Set aside smokers from both groups we found statistically very significantly elevated levels of sTREM-1 in BALF of PS patients. Concentrations of BALF sTREM-1 in PS non-smokers were more than four times higher than in non-smokers in the control ILD group $(p=0.001)$ (Tab. 4). Low levels of sTREM-1 in BALF of ILD non-smokers suggest low myeloid cells activation through TREM-1 receptor in the lungs. Our results are in agreement with findings of other authors whose results show rather low activity of TREM-1 molecule in ILD (16). Comparing ILD smokers with non-smokers statistically significantly higher levels of BALF sTREM-1 were found in ILD patients smokers ( $\mathrm{p}=0.0019)$ (Tab. 4).

In the next step we wanted to test the association of sTREM-1 concentrations in BALF with the presence of inflammation. As high concentrations of sTREM-1 point to inflammatory process amplification, we compared sTREM-1 levels in patients with and without hilar and/or mediastinal lymphadenopathy (LAP), regardless of the diagnosis. In BALF of patients with LAP, higher levels of sTREM-1 were detected comparing with patients without LAP $(p=0.0064)$ (Tab. 5). Moreover, patients with at least 3 elevated inflammatory markers (total proteins, IgG, IgA, IgM, C3 complement, alpha-2 macroglobulin) in BALF compared to patients without elevated inflammatory markers showed statistically significantly higher levels of sTREM-1 in BALF ( $p=0.042)$, regardless of the diagnosis (Tab. 5).

Comparing both investigated groups, the group of PS patients had statistically significantly higher percentage of all lymphocytes $(\mathrm{p}=0.0001)$, higher percentage of $\mathrm{CD} 3^{+} \mathrm{T}$ cells $(\mathrm{p}=0.0052)$, mainly $\mathrm{CD}^{+}$helper T cells $(\mathrm{p}<0.0001)$ and $\mathrm{CD}^{+} / \mathrm{CD}^{+}$ratio $\left.\mathrm{p}<0.0001\right)$ (Tab. 7) that are typical diagnostic markers for PS. These results show that laboratory findings in our PS group of patients are typical for sarcoidosis and that our selection of PS patients was correct.

The aim of our study was to test the hypothesis that the preferential expression of TREM-1 in PS is due to the presence of an infection what was documented in the last years, compared to other ILD of non-infectious origin. A study analyzing TREM-1 receptor in BALF in patients with pulmonary sarcoidosis has not been published yet. Pulmonary sarcoidosis characterized by significant lymphadenopathy, lymphocyte alveolitis with Thelper lymphocyte predominance (unlike other ILD) suggests significant macrophage activation and enhanced antigen presentation.

Our results show that sTREM-1 could be a good inflammatory marker only when tested locally in BALF and could help in diagnosis a monitoring of PS patients. As sTREM-1 is cleaved away from myeloid cell surfaces during their activation, detection of
STREM-1 in BALF indirectly point to myeloid cells activation in the lungs and helps to complete information about the number of myeloid cells commonly determined in BALF as diagnostic marker with additional information concerning the intensity of their activation. The results of our analysis show that TREM-1 molecule plays an important role in the pathogenesis of PS.

Increase of TREM-1 molecules (membrane and soluble form) is characteristic for infections caused by extracellular pathogenic microorganisms. Etiology of sarcoidosis, however, has been for decades associated with the presence of intracellular pathogens. This discrepancy can be explained by recent studies of Terčelj et al $(23,24)$, and the multicentric ACCESS (A Case Control Etiologic Study of Sarcoidosis) study (27) that have shown that potential etiologic agent for pulmonary sarcoidosis development is represented by fungi belonging to extracellular pathogens.

Our results are thus indirectly consistent with the results of these studies and support the opinion that extracellular pathogens play an important role in the etiology of sarcoidosis and the infection (chronic and/or subclinical) should be actively sought in sarcoidosis patients. As none of our PS patients had positive cultivation for commonly tested aerobic, anaerobic bacteria and mycobacterium tuberculosis, the active detection of mold infection should also be introduced to PS diagnosis.

Fungal glucans can bind to PRR receptors (e.g. dectin 1) on myeloid cells, activate them and this activation can be further enhanced by signalling through TREM-1 receptor. In fact, the involvement of this receptor was also described in Aspergillus granuloma in the liver and chronic Aspergillus asthma in mice (28). All PS patients enrolled in our study were in stage I and II of the disease. Further study will be necessary to determine the levels of sTREM-1 in a larger cohort of patients and in chronic forms of the diseases associated with fibrotic alteration of the lung interstitium.

Further studies are needed to evaluate different subgroups of ILD. For example, there was no patient with farmer's lung disease in our study. Conversely, high values of BALF sTREM-1 in ILD smokers indicate an increased activation of myeloid cells in the lungs. The explanation could be that the group of smokers includes 4 patients with COPD (44\%), (the main risk factor for COPD is smoking) and two patients with bullous emphysema. Our findings agree with previous COPD studies (11) and also with knowledge of the pathogenesis of COPD and emphysema, where myeloid cells are known to be involved in the lung tissue destruction.

\section{Conclusion}

sTREM-1 molecule is readily determinable inflammatory marker in BALF. Our results show elevated levels of inflammatory marker sTREM-1 in BALF, however not in plasma of patients with PS. Our results show that sTREM-1 could be a good inflammatory marker only when tested locally in BALF and could help in diagnosis and monitoring of PS patients. Higher concentrations of sTREM-1 were detected in BALF of patients with lymphadenopathy and those with elevated inflammatory markers in BALF. Moreover, detection of sTREM-1 in BALF indirectly points to myeloid cells activation in the lungs and helps to complete the informa- 
tion about the number of myeloid cells commonly determined in BALF as diagnostic marker with additional information concerning the intensity of their activation. From our results and the current knowledge we can assume that an active search for a chronic and/ /or subclinical infection (e.g. mold) in PS patients would be needed.

\section{References}

1. Bouchon A, Dietrich J, Colonna M. Cutting edge: inflammatory responses can be triggered by TREM-1, a novel receptor expressed on neutrophils and monocytes. J Immunol 2000; 164 (10): 4991-4995.

2. Bleharski JR, Kiessler V, Buonsanti C, Sieling PA, Stenger S, Colonna M, Modlin RL. A role for triggering receptor expressed on myeloid cells-1 in host defense during the early-induced and adaptive phases of the immune response. J Immunol 2003; 170 (7): 3812-3818.

3. Netea MG, Azam T, Ferwerda G, Girardin SE, Kim SH, Dinarello CA. Triggering receptor expressed on myeloid cells-1 (TREM-1) amplifies the signals induced by the NACHT-LRR (NLR) pattern recognition receptors. J Leukoc Biol 2006; 80 (6): 1454-1461.

4. Radsak MP, Salih HR, Rammensee HG, Schild H. Triggering receptor expressed on myeloid cells-1 in neutrophil inflammatory responses: differential regulation of activation and survival. J Immunol 2004; 172 (8): 4956-4963.

5. Gibot S, Buonsanti C, Massin F et al. Modulation of the triggering receptor expressed on the myeloid cell type 1 pathway in murine septic shock. Infect Immun 2006; 74 (5): 2823-2830.

6. Haselmayer P, Grosse-Hovest L, Landenberg P, Schild H, Radsak MP. TREM-1 ligand expression on platelets enhances neutrophil activation. Blood 2007; 110 (3): 1029-1035.

7. El Mezayen R, EI Gazzar M, Seeds MC, McCall CE, Dreskin SC, Nicolls MR. Endogenous signals released from necrotic cells augment inflammatory responses to bacterial endotoxin. Immunol Lett 2007; 111 (1): 36-44.

8. Gomez-Pina V, Soares-Schanoski A, Rodriguez-Rojas A et al. Metalloproteinases Shed TREM-1 Ectodomain from Lipopolysaccharide-Stimulated Human Monocytes. J Immunol 2007; 179 (6): 4065-4073.

9. Gibot S, Massin F. Soluble form of the triggering receptor expressed on myeloid cells 1: an anti-inflammatory mediator? Intensive Care Med 2006; 32 (2): 185-187.

10. Gibot S, Cravoisy A, Kolopp-Sarda MN, Bene MC, Faure G, Bollaert PE, Levy B. Time-course of sTREM (soluble triggering receptor expressed on myeloid cells)-1, procalcitonin, and C-reactive protein plasma concentrations during sepsis. Crit Care Med 2005; 33 (4): 792-796.

11. Radsak MP, Taube C, Haselmayer P et al. Soluble Triggering Receptor Expressed on Myeloid Cells 1 Is Released in Patients with Stable Chronic Obstructive Pulmonary Disease. Clin Dev Immunol 2007; 2007: 52040.

12. Tzivras M, Koussoulas V, Giamarellos-Bourboulis EJ et al. Role of soluble triggering receptor expressed on myeloid cells in inflammatory bowel disease. World J Gastroenterol 2006; 12 (21): 3416-3419.

13. Huang LY, Shi HZ, Liang QL, Wu YB, Qin XJ, Chen YQ. Expression of soluble triggering receptor expression on myeloid cells-1 in pleural effusion. Chin Med J 2008; 121 (17): 1656-1661.
14. Chan MC, Chang KM, Chao WC, Lin LY, Kuo BI, Hsu JY, Wu CL. Evaluation of a new inflammatory molecule (triggering receptor expressed on myeloid cells-1) in the diagnosis of pleural effusion. Respirology 2007; 12 (3): 333-338.

15. Menon R, Fortunato SJ. Induction of triggering receptors of myeloid cell (TREM-1) expression in fetal membranes and higher concentration of soluble TREM-1 in amniotic fluid with spontaneous preterm birth. Reprod Sci 2008; 15 (8): 825-830.

16. Richeldi L, Mariani M, Losi M et al. Triggering receptor expressed on myeloid cells: role in the diagnosis of lung infections. Eur Respir J 2004; 24 (2): 247-250.

17. Determann RM, Millo JL, Gibot $\mathbf{S}$ et al. Serial changes in soluble triggering receptor expressed on myeloid cells in the lung during development of ventilator-associated pneumonia. Intensive Care Med 2005; 31 (11): $1495-1500$.

18. Miao Y, Liu ZJ, Gong JP, Wei SD, Xu FL, Chen ZZ. Expression of human triggering receptor expressed on myeloid cells 1 in peripheral blood mononuclear cells of patients with acute obstructive suppurative cholangitis . Nan Fang Yi Ke Da Xue Xue Bao 2009; 29 (11): 2179-2181.

19. Liao R, Liu Z, Wei S, Xu F, Chen Z, Gong J. Triggering receptor in myeloid cells (TREM-1) specific expression in peripheral blood mononuclear cells of sepsis patients with acute cholangitis. Inflammation 2009; 32 (3): 182-190.

20. Ferat-Osorio E, Wong-Baeza I, Esquivel-Callejas $\mathbf{N}$ et al. Triggering receptor expressed on myeloid cells-1 expression on monocytes is associated with inflammation but not with infection in acute pancreatitis. Crit Care 2009; 13 (3): R69.

21. American Thoracic Society/European Respiratory Society. Joint Statement of the and the World Association of Sarcoidosis and Other Granulomatous Disorders (WASOG). Am J Respir Crit Care Med 1999; 160:736-755.

22. Jay H, Ryu MD, Craig $\mathbf{E}$ et al. Diagnosis of Interstitial Lung Diseases. Mayo Clin Proc 2007; 82 (8): 976-986.

23. Terčelj M, Stopinšek S, Ihan A, Salobir B, Simčič S, Wraber B, Rylander R. In vitro and in vivo reactivity to fungal cell wall agents in sarcoidosis. Clin Exp Immunol 2011; 166 (1): 87-93.

24. Terčelj M, Salobir B, Harlander M, Rylander R. Fungal exposure in homes of patients with sarcoidosis - an environmental exposure study. Environ Health 2011; 10: 8.

25. Gibot S, Cravoisy A, Levy B, Bene MC, Faure G, Bollaert PE. Soluble triggering receptor expressed on myeloid cells and the diagnosis of pneumonia. N Engl J Med 2004; 350 (5): 45-48.

26. Liu CL, Hsieh WY, Wu CL, Kuo HT, Lu YT. Triggering receptor expressed on myeloid cells-1 in pleural effusions: a marker of inflammatory disease. Respir Med 2007; 101 (5): 903-909.

27. Newman LS, Rose CS, Bresnitz EA et al. ACCESS Research Group. A case control etiologic study of sarcoidosis: environmental and occupational risk factors. Am J Respir Crit Care Med 2004; 170 (12): 1324-1330.

28. Buckland KF, Ramaprakash H, Murray LA et al. Triggering Receptor Expressed on Myeloid cells-1 (TREM-1) Modulates Immune Responses to Aspergillus fumigatus During Fungal Asthma in Mice. Immunol Invest 2011; 40 (7-8): 692-722. 